\title{
Giant Hepatic Cyst Treated by Laparoscopy
}

\author{
Medina Andrade Luis Angel MD ${ }^{1 *}$, Robles Méndez Hernández Alberto MD², Sánchez Dauth \\ Dulce María MD³ , Bastidas Meza Ezequiel Gabriel MD', Reus Muratalla Karen Stephanie \\ $\mathrm{MD}^{5}$, Vallejo Ramírez José Eduardo ${ }^{1}$ \\ ${ }^{1}$ General Surgery Department, Hospital General de Zona \#30 IMSS, Mexico City, Mexico \\ ${ }^{2}$ General Surgery Service, Universidad La Salle, Hospital Ángeles Metropolitano, México \\ ${ }^{3}$ General Surgery Service, Hospital Christus Muguerza del Parque, Mexico \\ ${ }^{4}$ General Surgery Service, Hospital de especialidades CMNSXXI, México \\ ${ }^{5}$ General Surgery Service, Hospital General $1^{\circ}$ de Octubre, ISSSTE
}

*Corresponding Author: Medina Andrade Luis Angel MD, General Surgery Department, Hospital General de Zona \#30 IMSS, Mexico City, Mexico,Email: buismedina_5@hotmail.com

\begin{abstract}
Background: Hepatic cysts have a prevalence of $4.6 \%$ in general population but only $5 \%$ become symptomatic and require surgical or non-surgical management.

Case Report: We present the case of a year-old female with a giant hepatic cyst in the right lobe. She underwent two previous percutaneous drainages with symptomatic relieve but recurrence after three and four months of procedure respectively. Her main symptom was abdominal pain, and when came to consultation the abdominal pain was constant and limiting her activities. One month before CT scan reported a giant hepatic cyst of nearly 2.7 l of liquid content rejecting all surrounding abdominal structures. A laparoscopicdrainage of $3.5 \mathrm{l}$ of cystic liquid and deroofing of cyst was performed without complications in 60 minutes of surgical time and patient could be discharged at $24 \mathrm{~h}$ without complications. At two months follow up she remains asymptomatic.
\end{abstract}

Conclusion: Laparoscopic deroofing of hepatic cyst is an excellent technique that offers all the advantages of minimally invasive approach, with less risk of recurrence to the patient and could be performed with excellent surgical times by highly trained surgeons.

Keywords: Giant hepatic cyst, hepatic cyst, advanced laparoscopy

\section{INTRODUCTION}

Hepatic cyst is generally a benign lesion founded incidentally during imaging studies for other pathologies. Their prevalence is 4.5 to $7 \%$ but is only treated when the patient present symptomatology, in general related with a big size and compression of other abdominal structures ${ }^{1}$. Giant abdominal cyst is considered when bigger than $6 \mathrm{~cm}$ in some reports, but we presented one case of a cyst of $23 \mathrm{~cm}$ of diameter and 3.5 liters of content treated laparoscopic ally.

\section{CASE RePort}

We present the case of a 42 year-old female with a giant hepatic cyst in the right lobe. She didn't referred other pathologies, allergies or previous surgeries. She underwent two previous percutaneous drainages with symptomatic relief but recurrence after three and four months of procedure respectively. Her main symptom was abdominal pain, and when came to consultation the abdominal pain was constant and limiting her activities. At physical exam she had a palpable tumor $10 \mathrm{~cm}$ under the right rib, with intense pain at superficial palpation, without acute abdomen signs. One month before a CT scan reported a giant hepatic cyst of nearly $2.7 \mathrm{l}$. of liquid content, rejecting all surrounding abdominal structures (Fig. 1). A laparoscopy was performed and giant hepatic cyst identified (Fig. 2), it was functioned and 3.51 . of clear cystic liquid drained. The cyst was added to right diaphragm and deroofing was performed without complications in 60 minutes of total 
surgical time (Fig. 3). The cyst wall was extracted in a simple bag. Oral intake began 6 hours after surgery with discharge at $24 \mathrm{~h}$ without complications. After two months of follow up she remains asymptomatic.

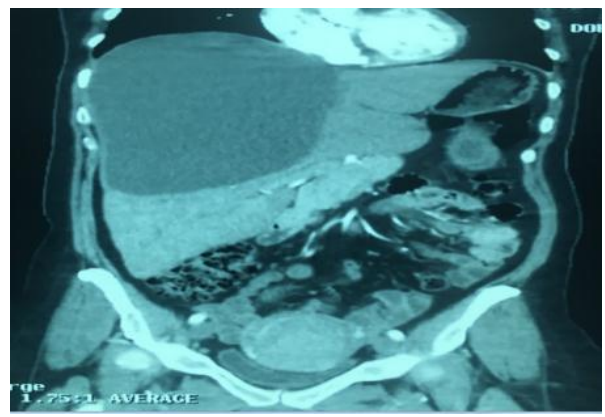

Figure1: $C T$ scan with giant hepatic cyst that descent hepatic parenchyma until right iliac spine.

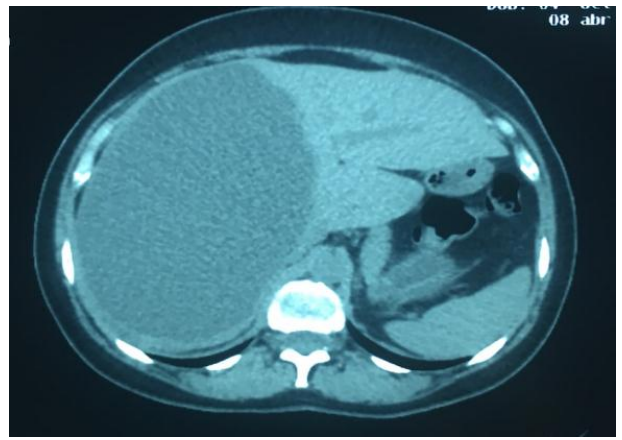

Figure2: CT scan with coronal approach that shows giant hepatic cyst with 3.3 liters of content.

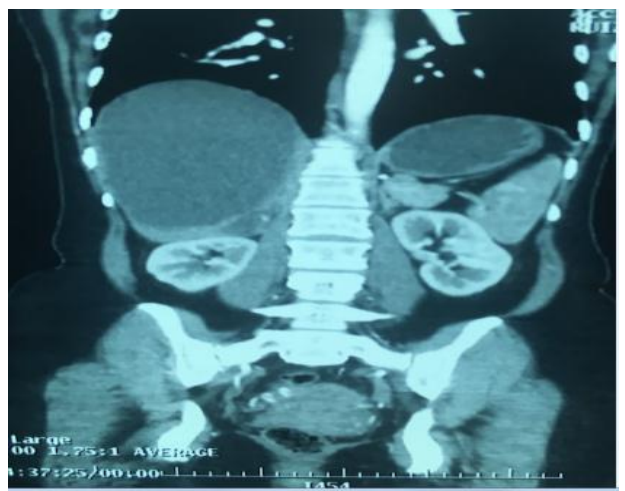

Figure3: $C T$ scan with sagital view confirming posterior extensión of giant hepatic cyst.

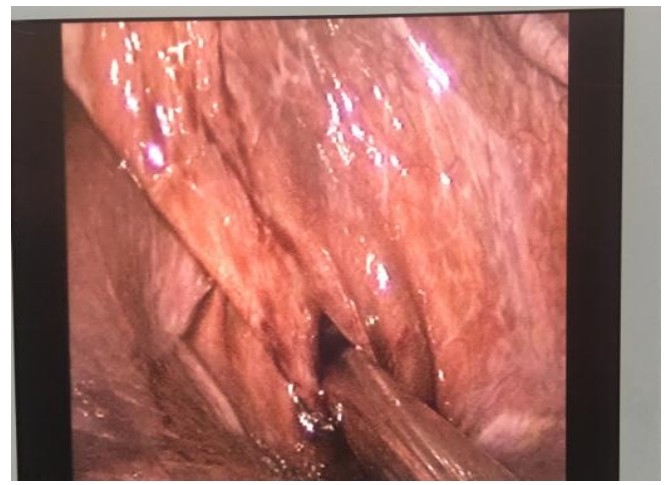

Figure4: Laparoscopic view of haptic cyst drainage of 3.5 liters of content.

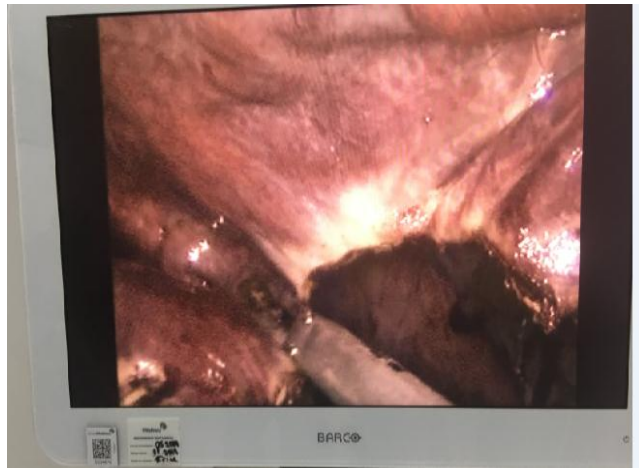

Figure5: Laparoscopic deroofig of giant hepatic cyst.

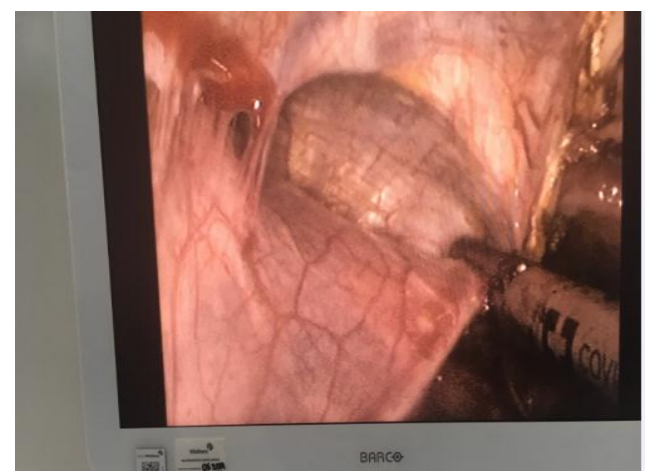

Figure6: Laparoscopic deroofing showing adherences to right hemidiafragm.

\section{DISCUSSION}

With exception of one previous report, and after an extensive research, this case is the largest giant hepatic cyst drained by laparoscopic technique at our knowledge. The presented patient presented a cyst that have been drained two previous times by ultrasound guided punction with evacuation of 2.7 liters of liquid in each, but as mentioned in the literature, this technique is associated with almost $100 \%$ of recurrence and is only an option for transient symptoms relief. Since the last CT scan to surgery the cyst increase in size to 3.51 , and could present spontaneous rupture by the size or with a minimal abdominal trauma leading to severe complications [1].

The prevalence of hepatic cyst in population is about 4.5 to $7 \%$, but the treatment of those is reserved for the ones that cause symptoms, accounting only for $5 \%$ of the cases. The main symptom on those cases is the abdominal pain associated with the size of the cyst, but other symptoms include jaundice for obstruction of biliary three, postprandial fulfill for compression of stomach, intestinal obstruction, bleeding, infection or spontaneous rupture with acute abdomen signs [1].

One of the main concerns during the approach is the image studies. CT scan is the gold standard 
in those cases, because it allows us to identify the general characteristics of the cyst, density, discard vascularity in case of tumoral lesions, septums, and increased density in abscess, biliary three involvements and the precise anatomic location to plannify the surgery. The cyst must be superficial in the hepatic surface to be punction and deroofed. Large cysts in the right posterior lobe have a high recurrence rate due to the intimate contact between liver and diaphragm, which blocks the continuous drainage of the deroofed cysts into the peritoneal cavity [2]. In cases of posterior location, the laparoscopic approach could not be performed and an open approach must be scheduled, until some author's reports good outcomes and advantages in difficult locations with robotic approach by Da Vinci [3].

The surgical approach have proved the better results along time, with minimal recurrence rate, and for this reason the ultrasound guided drain is almost discarded by the previous mentioned facts. Between the surgical techniques the open deroofing of cyst was first performed in 1968, and laparoscopic approach reported in 1991, developing new techniques according with new available technology and some cases of robotic assisted and single incision approaches successfully performed, some of the $\mathrm{m}$ with advantages and disadvantages but in general progressing to better results and less morbidity for patients $[1,4,5]$. Above the incisions the general approach is the same, trying to deroofe the hepatic cyst Surface previous aspiration of content, to resect all the cyst Surface near the union to the hepatic parenchyma to avoid recurrence, confirm the hemostasis and in some techniques use a material to cover the remaining cyst Surface over the liver, with glue or omentum, almost those techniques have not demonstrated improvement in results. The previous surgical approaches by laparoscopy reports surgical times between 90 to 230 minutes and hospital stay of one to three days. With actually enhanced recovery after surgery techniques we know that surgical time and reduced pneumoperitoneum is an important factor to reduce postoperative pain, nausea and hospital stay. In our case we complete the surgery in only 60 minutes which allow us to use minimum doses of analgesic and discharge uneventfully 24 hours after surgery although it was a case with one of the largest giant hepatic cysts reported in the literature.

\section{Conclusion}

Laparoscopic deroofing of hepatic cyst is an excellent technique that offers all the advantages of minimally invasive approach and since 1991 have been confirmed as the election technique to minimize complications and recurrence. It could be performed by highly trained surgeons with multiport or single port approaches with excellent results and patient's satisfaction, which converts it in the election technique for this pathology resolution.

\section{REFERENCES}

[1] Peter E. Fischer and Guy R. Voeller. Laparoscopic Management of a Giant Hepatic Cyst with Fibrin Glue Fixation of the Omentum. Surg Laparosc Endosc Percutan Tech 2011; 21:e273-e274.

[2] Chan Joong Choi, Young Hoon Kim, Young HoonRoh, GhapJoong Jung, et. al. Management of giant hepatic cysts in the laparoscopic era. J Korean SurgSoc2013; 85:116-122.

[3] Carolijn L. M. A., I. Quintus Molenaar, Inne H. M. Borel Rinkes, and Jeroen Hagendoorn. Robotassisted Laparoscopic Fenestration of Giant Hepatic Cysts. SurgLaparosc EndoscPercutan Tech 2015; 25:e163-e165.

[4] Takeshi Gocho, Takeyuki Misawa, Fumitake Suzuki, Ryusuke Ito, et. al. Single-incision laparoscopic surgery for giant hepatic cyst. Asian J EndoscSurg6 (2013) 237-240.

[5] Giovanni Dapri, Matteo Barabino, Pietro Carnevali, Ion Surdeanu, et. al. Single-Access Transumbilical Laparoscopic Unroofing of a Giant Hepatic Cyst Using Reusable Instruments. JSLS (2012)16:296-300.

Citation: Medina Andrade Luis Angel MD, Robles Méndez Hernández Alberto MD, et.al, Giant Hepatic Cyst Treated by Laparoscopy. ARC Journal of Surgery.2019; 5(3):1-3. DOI: http://dx.doi.org/10.20431/2455$572 X .0503001$

Copyright: ( $) 2019$ Authors. This is an open-access article distributed under the terms of the Creative Commons Attribution License, which permits unrestricted use, distribution, and reproduction in any medium, provided the original author and source are credited. 